\title{
Different Approaches of Bio-control Agents for Controlling Root Rot Incidence of Some Vegetables under Greenhouse Conditions
}

\author{
M. M. Abdel-Kader*, Nehal S. El-Mougy, M. DE. Aly, Lashin \\ Plant Pathology Department, National Research Centre, Giza, Egypt
}

\begin{abstract}
Different approaches of some antagonistic fungal, bacterial and yeast agents applied as seed treatment or soil drench was evaluated against various soil-borne pathogens causing vegetables root rot disease under greenhouse conditions. The tested pathogenic fungi were Alternaria solani Fusarium solani, F. oxysporum, Rhizoctonia solani, Sclerotium rolfsii, Macrophomina phaseolina and Pythium sp., meanwhile the tested bio-agents were Trichoderma harzianum, T. Viride and Bacillus subtilis, Pseudomonas flourescens and Sacchromyces serivisae. Significant reduction in the disease incidence was observed in bio-agent treatments in comparison with untreated control. Root rot incidence, at pre-emergence stage, significant effect was observed in bio-agent treatments as seed soaking comparing with soil drench treatment. The treated seeds showed a protective effect for seeds germination against the invasion by soil-borne pathogenic fungi. Meanwhile, soil drenched with different bio-agents showed more efficacy for reducing root rot incidence at post-emergence growth stage of tested vegetables, Cucumber, Cantaloupe, Tomato and Pepper. The obtained results revealed that the antagonist T. harzianum showed significant superior effect to reduce diseases incidence followed by B. subtilis. Also, the antagonists $T$. viride and $P$. fluorescence occupied significantly the second degree for reducing root rot incidence. The treatment with S. serevisiae had the lowest effect on disease incidence, although it significantly lesser than check control treatment. The present study demonstrates that application of bio-agents as seed treatment and soil drench may be useful for controlling root rot disease in field.
\end{abstract}

Keywords Root Rot, Seed Dressing, Soil Drench, Bio-Agents, Cucumber, Cantaloupe, Tomato, Pepper

\section{Introduction}

Vegetable crops are grown worldwide as a source of nutrients and fiber in the human diet. Fungal plant pathogens can cause devastation in these crops under appropriate environmental conditions. Vegetable producers confronted with the challenges of managing fungal pathogens have the opportunity to use fungi, bacteria and yeasts as biological control agents. Several commercially available products have shown significant disease reduction through various mechanisms to reduce pathogen development and disease. Plant diseases need to be controlled to maintain the quality and abundance of food, feed, and fiber produced by growers around the world. Different approaches may be used to prevent, mitigate or control plant diseases. Beyond good agronomic and horticultural practices, growers often rely heavily on chemical fertilizers and pesticides. Such inputs to agriculture have contributed significantly to the spectacular

* Corresponding author:

mokh_nrc@yahoo.com (M. M. Abdel-Kader)

Published online at http://journal.sapub.org/ijaf

Copyright (C) 2012 Scientific \& Academic Publishing. All Rights Reserved provements in crop productivity and quality over the past 100 years. However, the environmental pollution caused by excessive use and misuse of agrochemicals, as well as fear-mongering by some opponents of pesticides, has led to considerable changes in people's attitudes towards the use of pesticides in agriculture.

Today, there are strict regulations on chemical pesticide use, and there is political pressure to remove the most hazardous chemicals from the market. Additionally, the spread of plant diseases in natural ecosystems may preclude successful application of chemicals, because of the scale to which such applications might have to be applied. Consequently, some pest management researchers have focused their efforts on developing alternative inputs to synthetic chemicals for controlling pests and diseases. Among these alternatives are those referred to as biological control. The application of biological controls using antagonistic microorganisms has proved to be successful for controlling various plant diseases in many countries[1]. However, this is not an easy method, and it is costly to apply; however it can serve as the best control measure under greenhouse conditions. Trichoderma harzianum introduced to the soil, was able to reduce root rot incidence of faba bean plants significantly 
more than the fungicide Rizolex-T[2]. In recent years, several attempts have been made to overcome this obstacle by applying antagonistic microorganisms. Trichoderma spp. are well documented as effective biological control agents of plant diseases caused by soilborne fungi[3-5]. Many investigators[6-8] observed that the application of wheat bran colonised by $T$. harzianum to soil infested with $R$. solani and $S$. rolfsii, reduced the incidence of root diseases caused by these pathogens.

As for antagonistic bacteria, [9] found that seed treatment with Bacillus spp. was actively controlled three fungal root diseases of wheat. Also, Pseudomonas cepacia or Pseudomonas fluorescens applied to pea seeds act as biological control agent against Pythium damping-off and Aphanomyces root rot and was able to reduce diseases incidence $[10,11]$. Considerable researches has been done to investigate antagonistic microbes for use in seed treatments as reported by several workers[12-15]. In this regard, many crops are susceptible to seed and seedling root rot caused by soilborne fungi or by pathogens carried on the seed. Biological seed treatments may provide an alternative to chemical control of many soil and seed-borne pathogens. Bio-priming, a seed treatment system that integrates the biological and physiological aspects of disease control, involves coating the seed with fungal or bacterial biocontrol agents. Furthemore, soil drench with T. harzianum was significantly able to reduce the incidence of bean root rot of bean and pepper wilt diseases $[2,16]$.

The objective of the present study was to evaluate the different approaches activity of some antagonistic fungal and bacterial agents against vegetables root rot incidence when applied as seed treatment or soil drench under open greenhouse conditions.

\section{Materials and Methods}

\subsection{Plant Materials}

Seeds of Cucumber (cv. Alpha), Cantaloupe (cv. Yatherb 7), Tomato (cv. Castel Rock) and Pepper (cv. California) were used in the present study.

\subsection{Pathogenic Fungi}

The tested soilborne pathogenic fungi were Alternaria solani Fusarium solani, F. oxysporum, Rhizoctonia solani, Sclerotium rolfsii, Macrophomina phaseolina and Pythium sp. These fungi were isolated from various vegetables, i.e. Cucumber, Cantaloupe, Tomato and Pepper grown in plastic houses under protected cultivation system and showing root rot and or damping-off disease symptoms[17]. The isolated fungi proved their aggressive ability to induce root rot disease of those vegetables.

\subsection{Bio-agents}

The tested antagonistic fungi were Trichoderma harzianum, T. Viride and Bacillus subtilis, Pseudomonas flourescens and Sacchromyces serivisae. These antagonists were isolated from cucumber, cantaloupe, tomato and pepper grown in plastic houses under protected cultivation systems and showing root rot disease symptoms[17]. The present bio-agents proved their antagonistic ability against the above mentioned pathogens under in vitro conditions.

\subsection{Bio-agents and Pathogens Inocula Preparation}

The antagonistic bacteria (B. subtilis, P. flourescense) were grown on nutrient broth medium while yeast $(S$. serevisiae) was grown on NYDB medium[18]. All tested bacteria and yeast incubated in a rotary shaker at $200 \mathrm{rpm}$ for $24 \mathrm{~h}$ at $28 \pm 2^{\circ} \mathrm{C}$. The bacterial and yeast cells were harvested by centrifugation at $6,000 \mathrm{rpm}$ for $10 \mathrm{~min}$, washed twice with $0.05 \mathrm{M}$ phosphate buffer at $\mathrm{pH} 7.0$, and re- suspended in sterilized distilled water. The concentrations of both bacteria and yeast cells in the suspensions were adjusted to $1 \times 10^{5}-10^{6}$ cells per milliliter $(\mathrm{Cfu} / \mathrm{mL}$ ) with the aid of a haemacytometer slide. Meanwhile, antagonistic fungi were grown on PDA medium[18] incubated for $72 \mathrm{~h}$ at $25 \pm 2^{\circ} \mathrm{C}$. Fungal conidia were harvested by scraping the surface of the colonies with a spatula and transferred to sterilized distilled water and filtered through nylon mesh, then spore suspension was adjusted $1 \times 10^{4}-10^{5}$ spore per milliliter $(\mathrm{Cfu} / \mathrm{mL})$ with the aid of a haemacytometer slide.

On the other hand, Inocula of pathogenic fungi, Alternaria solani Fusarium solani, F. oxysporum, Rhizoctonia solani, Sclerotium rolfsii, Macrophomina phaseolina and Pythium sp. were individually grown on autoclaved sand barlly medium $(1: 1, \mathrm{v}: \mathrm{v}+40 \%$ water $)$ for two weeks at $25 \pm 1^{\circ} \mathrm{C}(\mathrm{Ab}-$ del-Kader, 1997). Soil infestation was carried out through amended individually with a pathogenic fungal inoculum $(5 \% \mathrm{w}: \mathrm{w})$ after[16], then mixed thoroughly to ensure equal distribution of the added inoculum. Infested soil was then filled in plastic pots (30-cm-diameter) and irrigated every second day for 1 week before sowing.

\subsection{Greenhouse Experiments}

Evaluation the efficacy of different approaches of bioagents against root rot incidence of Cucumber, Cantaloupe, Tomato and Pepper was carried out in pot experiment in the open greenhouse of Plant Pathology Dept., National Research Centre, Egypt. The evaluated bio-agents were applied either as seed soaking or soil drench.

Pathogens infested soil with the root rot pathogens inocula was used for this experiment. Seeds of Cucumber, Cantaloupe, Tomato and Pepper were soaked in individual fungal, bacterial or yeast sticky suspensions $(1 \mathrm{~mL}$ of Arabic gum/100 suspension) for one $\mathrm{hr}$, then picked up and air dried. Soaked seeds were sown as five seeds per pot, six pots per replicates in each treatment.

As for soil drench, the pathogens infested soils were re-infested individually with inocula of bio-agents either fungi (Trichoderma harzianum, T. viride, T. hamatum); bacteria (Bacillus subtilis, Pseudomonas fluorescence) or yeast (Saccharomyces serevisiae) relevant to the specific 
treatment. The fungal inocula were added to the soil at the ratio of $5 \%$ of soil weight, while bacterial inocula as liquid cultures $\left(3 \times 10^{6} \mathrm{cfu} / \mathrm{mL}\right)$ at the ratio of $100 \mathrm{ml} / \mathrm{cubic}$ foot of soil.

Another set of varied infested soils only with pathogens were filled in plastic pots (30- cm-diameter), was used for comparison check treatment. Another set of soil amended only with root rot pathogens $(5 \% \mathrm{w}$ :w) was Kept as control check treatment.

Surface sterilized seeds (using 3\% sodium hypochlorite for $5 \mathrm{~min}$, then picked up and air-dried) were used. Five seeds of each of Cucumber, Cantaloupe, Tomato and Pepper were planted in each pot and six replicated pots were used for each particular treatment. Percentage of root rot incidence at pre-emergence growth stage was recorded after two weeks from sowing date. Meanwhile, percentage of root rot incidence at post-emergence stage was calculated 45 days after seedling emergence. This experiment was repeated twice in order to confirm the obtained results.

Percentage of root rot incidence at the pre-emergence stage was calculated as the number of absent seedlings relative to the number of seeds sown. Meanwhile, the percentage of root rot incidence at the post-emergence stage was calculated as the number of diseased plants relative to the number of emerging seedlings. At the end of the experiment, survived plants were carefully pulled out from pots after being flooded with water in order to leave the root system undamaged. Plant roots showing rot lesions in addition to the visual root rot symptoms on the shoot system were considered diseased plants. Isolation from infected germinated seeds at the pre-emergence stage and infected plants at the post-emergence stage was carried out. Undeveloped, germinated seeds which were picked up from the soil, and the diseased plants were both washed and sterilized with 3\% sodium hypochlorite, then subjected to re-isolation in order to identify the causal pathogens.

Statistical analysis

All experiments were set up in a complete randomized design. One-way ANOVA was used to analyze differences between applied treatments and disease incidence. A general linear model option of the analysis system SAS[19] was used to perform the ANOVA. Duncan's multiple range test at $P<0.05$ level was used for means separation[20].

\section{Results and Discussion}

The efficacy of soaked Cucumber, Cantaloupe, Tomato and Pepper seeds or soil drench with the fungal and bacterial bio-agents against vegetables root rot incidence caused by some soil-borne pathogenic fungi was evaluated in a pot experiment using soil artificially infested with the pathogen under open greenhouse conditions.

The incidence of pre-, and post-emergence root rot are presented in Tables $(1,2$ \& Figs. 1, 2) for seed treatment application and Tables (3, 4 \& Figs 3, 4) for soil drench application, in respective order. Presented data show that the applied bio-gent treatments either as seed soaking or soil drench caused a significant effect on root rot incidence at both plant growth stages of grown vegetables comparing with control.

Regarding root rot incidence at pre-emergence stage, a significant effect was observed in bio-agents treatment as seed soaking comparing with soil drench treatment (Tables 1 and 3 \& Figs 1 and 3). The treated seeds showed a protective effect for seeds germination against the invasion by soil-borne pathogenic fungi. Data also revealed that the antagonist $T$. harzianum showed significant superior effect to reduce diseases incidence followed by $B$. subtilis. Also, the antagonists $T$. viride and $P$. fluorescence occupied significantly the second degree for reducing root rot incidence. The treatment with $S$. serevisiae had the lowest effect on disease incidence, although it significantly lesser than check control treatment. This observation was true with all vegetables tested. In this regards, the antagonistic fungi were more actively than the two antagonistic bacteria for reducing root rot incidence at pre-emergence stage of plant growth. Furthermore, data also showed that the lowest root rot incidence was recorded with Cantaloupe and cucumber followed by Tomato and Pepper plants. Data also revealed that the pathogenic fungi $S$. rolfsii, $R$. solani, F. solani, $F$. oxysporum, Pythium sp., M. phaseolina and S. sclerotiorum showed more response to antagonistic fungi and bacteria which reflected in recorded minimization of the root rot incidence.

At pre-emergence stage, Data in Table (1) showed that the recorded percentage of root rot incidence in seed soaking treatments in medium ranged between $25.5-28.8 \%$, 24.4$23.9 \%, 17.0-32.2 \%$ and $17.3-31.8 \%$ comparing with control treatment as $42.9,45.1,46.2$ and $42.9 \%$ for Cucumber, Cantaloupe, Tomato and Pepper, respectively. Meanwhile, the recorded percentage of root rot incidence in soil drench treatments in medium ranged between $27.7-45.8 \%, 25.1$ $42.9 \%, 24.7-44.4 \%$ and $25.1-43.6 \%$ comparing with control treatment as $50.7,50.3,51.0$ and $48.8 \%$ for grown vegetables in respective order.

Soil drenched with different bio-agents showed more efficacy for reducing root rot incidence at post-emergence growth stage of tested vegetables. In this concern, presented data in Tables (2 and 4) and Figs (2 and 4) revealed that percentage of root rot incidence highly reduced as 3.3, 11.4, 34.9 and $35.3 \%$ in soil drenched with $T$. harzianum comparing with $32.5,30.5,30.4$ and $30.9 \%$ in seed soaking treatment with the same fungus for Cucumber, Cantaloupe, Tomato and Pepper, respectively. Similar observations were also recorded in soil drench treatment with T. viride, B. subtilis, P. flourescens and $S$. serevisiae. Moreover, data in Tables (2 and 4) also showed that soil drench with T. harzianum caused the lowest significant reduction in root rot incidence followed by $T$. viride, B. subtilis, P. flourescens and $S$. serevisiae, respectively. Furthermore, T. harzianum and $B$. subtilis drenched soil showed interested results against root rot pathogens that complete reduction $(100 \%)$ in disease incidence was recorded for Cucumber and Cantaloupe in infested soil with the most tested pathogenic fungi comparing with $90-89.1 \%$ infection recorded in control check treatment. 
The present study shows that bio-agents applied as soil drench was the most favorite method of application resulted in the best control records. The obtained reduction in invaded vegetable plants with root rot pathogens may be attributed to the high accumulative inoculum potential of the introduced bio-agents into the root region, before sowing and throughout the growing season as well, where they are predicted to have a direct impact on already established pathogens population. Similar explanation was reported[2], who stated that, soil treatment with biocide $T$. harzianum showed better reduction in root rot incidence of bean followed by seed coating with the biocide. He added that, these differences could be due to the initial inoculum of $T$. harzianum introduced into the soil. Moreover, the high fungal population density introduced through soil treatment technique enables the fungus to adapt itself against environmental conditions[21] resulting in dominance of high population of the introduced fungi in the plant rhizosphere. The use of microorganisms that antagonize plant pathogens (biological control) is risk-free when it results in enhancement of resident antagonists. Moreover, the combination of such biological control agents (BCAs) with reduced levels of fungicide (integrated control) promotes a degree of disease suppression similar to that achieved with full fungicide treatment[22]. Moreover, the application of biological controls using antagonistic microorganisms, has proved to be successful for controlling various plant diseases in many countries [1,23-26].

Table 1. Effect of applying antagonistic bio-agents as seed dressing against vegetables root diseases at re-emergence growth stage caused by soil-borne pathogenic fungi under open greenhouse conditions

\begin{tabular}{|c|c|c|c|c|c|c|c|c|c|c|c|}
\hline \multirow[b]{3}{*}{ plant } & \multirow[b]{3}{*}{ Bio-agent } & \multicolumn{10}{|c|}{ Pre-emergence root diseases incidence (\%) } \\
\hline & & \multicolumn{10}{|c|}{ Soil-borne pathogenic fungi } \\
\hline & & $\begin{array}{c}\text { S. } \\
\text { rolfsii }\end{array}$ & $\begin{array}{c}\mathrm{R} . \\
\text { solani }\end{array}$ & $\begin{array}{c}\text { F. } \\
\text { solani }\end{array}$ & $\begin{array}{c}\mathrm{F} . \\
\text { Oxys- } \\
\text { porum }\end{array}$ & $\begin{array}{c}\text { S. } \\
\text { Sclero- } \\
\text { tiorum }\end{array}$ & $\begin{array}{c}\mathrm{S} . \\
\text { minor }\end{array}$ & $\begin{array}{c}\text { M. } \\
\text { phaseolina }\end{array}$ & $\begin{array}{c}\text { Pythium } \\
\text { sp. }\end{array}$ & $\begin{array}{c}\text { A. } \\
\text { solani }\end{array}$ & Mean \\
\hline \multirow{6}{*}{ Cucumber } & T. harzianum & $16.6 \mathrm{~h}$ & $20.0 \mathrm{~g}$ & $23.3 \mathrm{fg}$ & $26.6 \mathrm{f}$ & $30.0 \mathrm{e}$ & $26.6 \mathrm{f}$ & $30.0 \mathrm{e}$ & $30.0 \mathrm{e}$ & $26.6 \mathrm{f}$ & 25.5 \\
\hline & T. viride & $33.3 \mathrm{de}$ & $33.3 \mathrm{de}$ & $26.6 \mathrm{f}$ & $23.3 \mathrm{fg}$ & $33.3 \mathrm{de}$ & $30.0 \mathrm{e}$ & $26.6 \mathrm{f}$ & $30.0 \mathrm{e}$ & $23.3 \mathrm{fg}$ & 28.8 \\
\hline & B. subtilis & $13.3 \mathrm{~h}$ & $23.3 \mathrm{fg}$ & $26.6 \mathrm{f}$ & $20.0 \mathrm{~g}$ & $30.0 \mathrm{e}$ & $26.6 \mathrm{f}$ & $33.3 \mathrm{de}$ & 33.3 de & $26.6 \mathrm{f}$ & 25.8 \\
\hline & P. fluorescence & $30.0 \mathrm{e}$ & $33.3 \mathrm{de}$ & $30.0 \mathrm{e}$ & $23.3 \mathrm{fg}$ & $30.0 \mathrm{e}$ & $26.6 \mathrm{f}$ & $23.3 \mathrm{fg}$ & $33.3 \mathrm{de}$ & $30.0 \mathrm{e}$ & 28.8 \\
\hline & S. serivisae & $33.3 \mathrm{de}$ & $30.0 \mathrm{e}$ & $26.6 \mathrm{f}$ & $23.3 \mathrm{fg}$ & $23.3 \mathrm{fg}$ & $26.6 \mathrm{f}$ & $30.0 \mathrm{e}$ & $33.3 \mathrm{de}$ & $33.3 \mathrm{de}$ & 28.8 \\
\hline & Control & $46.6 \mathrm{bc}$ & $50.0 \mathrm{~b}$ & $46.6 \mathrm{bc}$ & $26.6 \mathrm{f}$ & $36.6 \mathrm{f}$ & $46.6 \mathrm{bc}$ & $43.3 \mathrm{c}$ & $56.6 \mathrm{ab}$ & $33.3 \mathrm{de}$ & 42.9 \\
\hline \multirow{6}{*}{ Cantaloupe } & T. harzianum & $13.3 \mathrm{~h}$ & $16.6 \mathrm{gh}$ & $26.6 \mathrm{f}$ & $23.3 \mathrm{fg}$ & $26.6 \mathrm{f}$ & $26.6 \mathrm{f}$ & $26.6 \mathrm{f}$ & $30.0 \mathrm{e}$ & $30.0 \mathrm{e}$ & 24.4 \\
\hline & $\mathrm{T}$. viride & $33.3 \mathrm{de}$ & $33.3 \mathrm{de}$ & $30.0 \mathrm{e}$ & $26.6 \mathrm{f}$ & $33.3 \mathrm{de}$ & $33.3 \mathrm{de}$ & $30.0 \mathrm{e}$ & $33.3 \mathrm{de}$ & $26.6 \mathrm{f}$ & 31.0 \\
\hline & B. subtilis & $16.6 \mathrm{gh}$ & $26.6 \mathrm{f}$ & $23.3 \mathrm{fg}$ & $33.3 \mathrm{de}$ & $26.6 \mathrm{f}$ & $30.0 \mathrm{e}$ & $30.0 \mathrm{e}$ & $30.0 \mathrm{e}$ & $23.3 \mathrm{fg}$ & 26.6 \\
\hline & P. fluorescence & $33.3 \mathrm{de}$ & $30.0 \mathrm{e}$ & $46.6 \mathrm{bc}$ & $20.0 \mathrm{~g}$ & $20.0 \mathrm{~g}$ & $40.0 \mathrm{~cd}$ & $33.3 \mathrm{de}$ & $30.0 \mathrm{e}$ & $33.3 \mathrm{de}$ & 31.8 \\
\hline & S. serivisae & $33.3 \mathrm{de}$ & $33.3 \mathrm{de}$ & $30.0 \mathrm{e}$ & $36.6 \mathrm{~d}$ & $33.3 \mathrm{de}$ & $30.0 \mathrm{e}$ & $33.3 \mathrm{de}$ & $33.3 \mathrm{de}$ & $33.3 \mathrm{de}$ & 32.9 \\
\hline & Control & $60.0 \mathrm{a}$ & $40.0 \mathrm{~cd}$ & $50.0 \mathrm{~b}$ & $36.6 \mathrm{~d}$ & $36.6 \mathrm{e}$ & $50.0 \mathrm{~b}$ & $46.6 \mathrm{bc}$ & $53.3 \mathrm{~b}$ & $33.3 \mathrm{de}$ & 45.1 \\
\hline \multirow{6}{*}{ Tomato } & T. harzianum & $16.6 \mathrm{gh}$ & $13.3 \mathrm{~d}$ & $16.6 \mathrm{gh}$ & $20.0 \mathrm{~g}$ & $23.3 \mathrm{fg}$ & $13.3 \mathrm{~h}$ & $16.6 \mathrm{gh}$ & $16.6 \mathrm{gh}$ & $20.0 \mathrm{~g}$ & 17.3 \\
\hline & T. viride & $33.3 \mathrm{de}$ & $33.3 \mathrm{de}$ & $30.0 \mathrm{e}$ & $30.0 \mathrm{e}$ & $33.3 \mathrm{de}$ & $30.0 \mathrm{e}$ & $33.3 \mathrm{de}$ & $30.0 \mathrm{e}$ & $33.3 \mathrm{de}$ & 31.8 \\
\hline & B. subtilis & $13.3 \mathrm{~h}$ & $16.6 \mathrm{gh}$ & $20.0 \mathrm{~g}$ & $23.3 \mathrm{fg}$ & $13.3 \mathrm{~h}$ & $16.6 \mathrm{gh}$ & $13.3 \mathrm{~h}$ & $16.6 \mathrm{gh}$ & $20.0 \mathrm{~g}$ & 17.0 \\
\hline & P. fluorescence & $33.3 \mathrm{de}$ & $33.3 \mathrm{de}$ & $30.0 \mathrm{e}$ & $33.3 \mathrm{de}$ & $30.0 \mathrm{e}$ & $33.3 \mathrm{de}$ & $30.0 \mathrm{e}$ & $33.3 \mathrm{de}$ & $33.3 \mathrm{de}$ & 32.2 \\
\hline & S. serivisae & $33.3 \mathrm{de}$ & $30.0 \mathrm{e}$ & $33.3 \mathrm{de}$ & $33.3 \mathrm{de}$ & $33.3 \mathrm{de}$ & $30.0 \mathrm{e}$ & $30.0 \mathrm{e}$ & $33.3 \mathrm{de}$ & $33.3 \mathrm{de}$ & 32.2 \\
\hline & Control & $50.0 \mathrm{~b}$ & $46.6 \mathrm{bc}$ & $60.0 \mathrm{a}$ & $33.3 \mathrm{de}$ & $40.0 \mathrm{~cd}$ & $53.3 \mathrm{~b}$ & $40.0 \mathrm{~cd}$ & $60.0 \mathrm{a}$ & $33.3 \mathrm{de}$ & 46.2 \\
\hline \multirow{6}{*}{ Pepper } & T. harzianum & $13.3 \mathrm{~h}$ & $16.6 \mathrm{gh}$ & $20.0 \mathrm{~g}$ & $23.3 \mathrm{fg}$ & $26.6 \mathrm{f}$ & $16.6 \mathrm{gh}$ & $13.3 \mathrm{~h}$ & $20.0 \mathrm{~g}$ & $23.3 \mathrm{fg}$ & 19.2 \\
\hline & T. viride & $33.3 \mathrm{de}$ & $30.0 \mathrm{e}$ & $33.3 \mathrm{de}$ & $26.6 \mathrm{f}$ & $30.0 \mathrm{e}$ & $33.3 \mathrm{de}$ & $36.6 \mathrm{~d}$ & $33.3 \mathrm{de}$ & $30.0 \mathrm{e}$ & 31.8 \\
\hline & B. subtilis & $10.0 \mathrm{hi}$ & $13.3 \mathrm{~h}$ & $23.3 \mathrm{fg}$ & $20.0 \mathrm{~g}$ & $16.6 \mathrm{gh}$ & $13.3 \mathrm{~h}$ & $16.6 \mathrm{gh}$ & $20.0 \mathrm{~g}$ & $23.3 \mathrm{fg}$ & 17.3 \\
\hline & P. fluorescence & $33.3 \mathrm{de}$ & $30.0 \mathrm{e}$ & $33.3 \mathrm{de}$ & $30.0 \mathrm{e}$ & $33.3 \mathrm{de}$ & $30.0 \mathrm{e}$ & $33.3 \mathrm{de}$ & $30.0 \mathrm{e}$ & $33.3 \mathrm{de}$ & 31.8 \\
\hline & S. serivisae & $33.3 \mathrm{de}$ & $26.6 \mathrm{f}$ & $30.0 \mathrm{e}$ & $33.3 \mathrm{de}$ & $30.0 \mathrm{e}$ & $26.6 \mathrm{f}$ & $26.6 \mathrm{f}$ & $30.0 \mathrm{e}$ & $33.3 \mathrm{de}$ & 29.9 \\
\hline & Control & $53.3 \mathrm{~b}$ & $43.3 \mathrm{c}$ & $40.0 \mathrm{~cd}$ & $33.3 \mathrm{de}$ & $46.6 \mathrm{bc}$ & $40.0 \mathrm{~cd}$ & $50.0 \mathrm{~b}$ & $46.6 \mathrm{bc}$ & $33.3 \mathrm{de}$ & 42.9 \\
\hline
\end{tabular}

Mean values within columns followed by the same letter are not significantly different $(\mathrm{P} \leq 0.05)$ 
Table 2. Effect of Effect of applying antagonistic bio-agents as seed dressing against vegetables root diseases at post-emergence growth stage caused by soil-borne pathogenic fungi under open greenhouse conditions

\begin{tabular}{|c|c|c|c|c|c|c|c|c|c|c|c|}
\hline \multirow[b]{3}{*}{ plant } & \multirow[b]{3}{*}{ Bio-agent } & \multicolumn{10}{|c|}{ Pre-emergence root diseases incidence (\%) } \\
\hline & & \multicolumn{10}{|c|}{ Soil-borne pathogenic fungi } \\
\hline & & $\begin{array}{l}S . \\
\text { rolfsii }\end{array}$ & R. solani & $\begin{array}{c}F . \\
\text { solani }\end{array}$ & $\begin{array}{c}F . \\
\text { ox- } \\
\text { ysporum }\end{array}$ & $\begin{array}{c}S . \\
\text { scle- } \\
\text { rotiorum }\end{array}$ & $\begin{array}{c}S . \\
\text { minor }\end{array}$ & $\begin{array}{c}\text { M. } \\
\text { phaseo- } \\
\text { lina }\end{array}$ & $\begin{array}{c}P y- \\
\text { thium } \\
\text { sp. }\end{array}$ & $\begin{array}{c}A . \\
\text { solani }\end{array}$ & Mean \\
\hline \multirow{6}{*}{ Cucumber } & T. harzianum & $40.0 \mathrm{hi}$ & $37.5 \mathrm{i}$ & $30.4 \mathrm{j}$ & $45.4 \mathrm{~h}$ & $28.5 \mathrm{jk}$ & $27.2 \mathrm{jk}$ & $33.3 \mathrm{j}$ & $28.5 \mathrm{jk}$ & $21.7 \mathrm{k}$ & 32.5 \\
\hline & T. viride & $45.0 \mathrm{~h}$ & $40.0 \mathrm{hi}$ & $40.9 \mathrm{hi}$ & $47.8 \mathrm{~h}$ & $50.0 \mathrm{fg}$ & $33.3 \mathrm{j}$ & $31.8 \mathrm{j}$ & $33.3 \mathrm{j}$ & $43.7 \mathrm{~h}$ & 40.6 \\
\hline & B. subtilis & $30.7 \mathrm{j}$ & $34.7 \mathrm{j}$ & $22.7 \mathrm{k}$ & $33.3 \mathrm{j}$ & 14.21 & $22.7 \mathrm{k}$ & $20.0 \mathrm{k}$ & $25.0 \mathrm{k}$ & 18.11 & 24.6 \\
\hline & P. fluorescence & $42.8 \mathrm{hi}$ & $35.0 \mathrm{i}$ & $33.3 \mathrm{j}$ & $40.0 \mathrm{hi}$ & $38.0 \mathrm{i}$ & $45.4 \mathrm{~h}$ & $52.1 \mathrm{fg}$ & $40.0 \mathrm{hi}$ & $33.3 \mathrm{j}$ & 39.9 \\
\hline & S. serivisae & $50.0 \mathrm{fg}$ & $47.6 \mathrm{~h}$ & $31.8 \mathrm{j}$ & $43.4 \mathrm{~h}$ & $39.1 \mathrm{i}$ & $31.8 \mathrm{j}$ & $33.3 \mathrm{j}$ & $50.0 \mathrm{fg}$ & $50.0 \mathrm{fg}$ & 41.8 \\
\hline & Control & $68.7 \mathrm{e}$ & $73.3 \mathrm{~d}$ & $62.5 \mathrm{e}$ & $81.8 \mathrm{c}$ & $89.4 \mathrm{c}$ & $93.7 \mathrm{~b}$ & $100 \mathrm{a}$ & $84.6 \mathrm{c}$ & $80.0 \mathrm{c}$ & 81.5 \\
\hline \multirow{6}{*}{ Cantaloupe } & T. harzianum & $38.4 \mathrm{i}$ & $36.0 \mathrm{i}$ & $31.8 \mathrm{j}$ & $34.7 \mathrm{j}$ & $27.2 \mathrm{jk}$ & $31.8 \mathrm{j}$ & $31.8 \mathrm{j}$ & $23.8 \mathrm{k}$ & 19.01 & 30.5 \\
\hline & T. viride & $40.0 \mathrm{hi}$ & $45.0 \mathrm{~h}$ & $33.3 \mathrm{j}$ & $50.0 \mathrm{fg}$ & $45.0 \mathrm{~h}$ & $35.0 \mathrm{j}$ & $33.3 \mathrm{j}$ & $40.0 \mathrm{hi}$ & $36.3 \mathrm{i}$ & 40.8 \\
\hline & B. subtilis & $24.0 \mathrm{k}$ & $27.2 \mathrm{jk}$ & $30.4 \mathrm{j}$ & $26.0 \mathrm{jk}$ & 13.61 & 14.21 & 19.01 & $23.8 \mathrm{k}$ & 17.31 & 21.7 \\
\hline & P. fluorescence & $45.0 \mathrm{~h}$ & $42.8 \mathrm{hi}$ & $50.0 \mathrm{fg}$ & $66.6 \mathrm{e}$ & $60.0 \mathrm{e}$ & $44.2 \mathrm{~h}$ & $40.0 \mathrm{hi}$ & $33.3 \mathrm{j}$ & $35.0 \mathrm{j}$ & 46.3 \\
\hline & S. serivisae & $45.0 \mathrm{~h}$ & $45.0 \mathrm{~h}$ & $47.6 \mathrm{~h}$ & $47.3 \mathrm{~h}$ & $40.0 \mathrm{hi}$ & $33.3 \mathrm{j}$ & $50.0 \mathrm{fg}$ & $40.0 \mathrm{hi}$ & $50.0 \mathrm{fg}$ & 44.2 \\
\hline & Control & $83.3 \mathrm{c}$ & $94.4 \mathrm{~b}$ & $73.3 \mathrm{~d}$ & $90.0 \mathrm{~b}$ & $94.7 \mathrm{~b}$ & $100 \mathrm{a}$ & $93.7 \mathrm{~b}$ & $92.8 \mathrm{~b}$ & $80.0 \mathrm{c}$ & 89.1 \\
\hline \multirow{6}{*}{ Tomato } & T. harzianum & $24.0 \mathrm{k}$ & $34.6 \mathrm{j}$ & $28.0 \mathrm{jk}$ & $29.1 \mathrm{jk}$ & $34.7 \mathrm{j}$ & $38.4 \mathrm{i}$ & $28.0 \mathrm{jk}$ & $32.0 \mathrm{j}$ & $25.0 \mathrm{k}$ & 30.4 \\
\hline & T. viride & $45.0 \mathrm{~h}$ & $50.0 \mathrm{fg}$ & $47.6 \mathrm{~h}$ & $42.8 \mathrm{hi}$ & $50.0 \mathrm{fg}$ & $47.6 \mathrm{~h}$ & $45.0 \mathrm{~h}$ & $57.1 \mathrm{f}$ & $50.0 \mathrm{fg}$ & 48.3 \\
\hline & B. subtilis & $30.7 \mathrm{j}$ & $28.0 \mathrm{jk}$ & $29.1 \mathrm{jk}$ & $26.0 \mathrm{jk}$ & $42.3 \mathrm{hi}$ & $32.0 \mathrm{j}$ & $34.6 \mathrm{j}$ & $28.0 \mathrm{jk}$ & $20.8 \mathrm{k}$ & 30.1 \\
\hline & P. fluorescence & $55.0 \mathrm{f}$ & $45.0 \mathrm{~h}$ & $47.6 \mathrm{~h}$ & $50.0 \mathrm{fg}$ & $52.3 \mathrm{fg}$ & $45.0 \mathrm{~h}$ & $47.6 \mathrm{~h}$ & $50.0 \mathrm{fg}$ & $50.0 \mathrm{fg}$ & 49.1 \\
\hline & S. serivisae & $50.0 \mathrm{fg}$ & $52.3 \mathrm{fg}$ & $45.0 \mathrm{~h}$ & $50.0 \mathrm{fg}$ & $45.0 \mathrm{~h}$ & $52.3 \mathrm{fg}$ & $47.6 \mathrm{~h}$ & $55.0 \mathrm{fg}$ & $50.0 \mathrm{fg}$ & 49.6 \\
\hline & Control & $60.0 \mathrm{e}$ & $68.7 \mathrm{e}$ & $91.6 \mathrm{~b}$ & $85.0 \mathrm{c}$ & $94.4 \mathrm{~b}$ & $100 \mathrm{a}$ & $100 \mathrm{a}$ & $91.6 \mathrm{~b}$ & $86.9 \mathrm{c}$ & 86.4 \\
\hline \multirow{6}{*}{ Pepper } & T. harzianum & $30.7 \mathrm{j}$ & $36.0 \mathrm{i}$ & $29.1 \mathrm{jk}$ & $21.7 \mathrm{k}$ & $27.2 \mathrm{jk}$ & $40.0 \mathrm{hi}$ & $34.6 \mathrm{j}$ & $33.3 \mathrm{j}$ & $26.0 \mathrm{jk}$ & 30.9 \\
\hline & T. viride & $40.0 \mathrm{hi}$ & $47.6 \mathrm{~h}$ & $50.0 \mathrm{fg}$ & $40.9 \mathrm{hi}$ & $47.6 \mathrm{~h}$ & $40.0 \mathrm{hi}$ & $47.3 \mathrm{~h}$ & $50.0 \mathrm{fg}$ & $47.6 \mathrm{~h}$ & 45.6 \\
\hline & B. subtilis & $37.0 \mathrm{i}$ & $34.6 \mathrm{j}$ & $30.4 \mathrm{j}$ & $33.3 \mathrm{j}$ & $40.0 \mathrm{hi}$ & $38.4 \mathrm{j}$ & $36.0 \mathrm{i}$ & $29.1 \mathrm{jk}$ & $21.7 \mathrm{k}$ & 33.3 \\
\hline & P. fluorescence & $50.0 \mathrm{f}$ & $52.3 \mathrm{fg}$ & $40.0 \mathrm{hi}$ & $47.6 \mathrm{~h}$ & $40.0 \mathrm{hi}$ & $52.3 \mathrm{fg}$ & $55.0 \mathrm{f}$ & $47.6 \mathrm{~h}$ & $50.0 \mathrm{fg}$ & 48.3 \\
\hline & S. serivisae & $45.0 \mathrm{~h}$ & $59.0 \mathrm{f}$ & $52.3 \mathrm{fg}$ & $45.0 \mathrm{~h}$ & $42.8 \mathrm{hi}$ & $54.5 \mathrm{~h}$ & $50.0 \mathrm{fg}$ & $52.0 \mathrm{fg}$ & $50.0 \mathrm{fg}$ & 50.0 \\
\hline & Control & $78.5 \mathrm{~d}$ & $88.2 \mathrm{c}$ & $72.2 \mathrm{~d}$ & $75.0 \mathrm{~d}$ & $100 \mathrm{a}$ & $94.4 \mathrm{~b}$ & $93.3 \mathrm{~b}$ & $93.7 \mathrm{~b}$ & $76.1 \mathrm{~d}$ & 85.7 \\
\hline
\end{tabular}

Mean values within columns followed by the same letter are not significantly different $(\mathrm{P} \leq 0.05)$.

Table 3. Effect of applying antagonistic bio-agents as soil treatment against vegetables root diseases at pre-emergence growth stage caused by soil-borne pathogenic fungi under open greenhouse conditions

\begin{tabular}{|c|c|c|c|c|c|c|c|c|c|c|c|}
\hline \multirow{3}{*}{ plant } & \multirow{3}{*}{ Bio-agent } & \multicolumn{10}{|c|}{ Pre-emergence root diseases incidence (\%) } \\
\hline & & \multicolumn{10}{|c|}{ Soil-borne pathogenic fungi } \\
\hline & & $\begin{array}{c}\mathrm{S} . \\
\text { rolfsii }\end{array}$ & $\begin{array}{c}\mathrm{R} . \\
\text { solani }\end{array}$ & $\begin{array}{c}\text { F. } \\
\text { solani }\end{array}$ & $\begin{array}{c}\text { F. ox- } \\
\text { ysporum }\end{array}$ & $\begin{array}{l}\text { S. scle- } \\
\text { rotiorum }\end{array}$ & $\begin{array}{c}\text { S. } \\
\text { minor }\end{array}$ & $\begin{array}{l}\text { M. phaseo- } \\
\text { lina }\end{array}$ & $\begin{array}{l}\text { Pythium } \\
\text { sp. }\end{array}$ & A. solani & Mean \\
\hline \multirow{6}{*}{$\begin{array}{l}\text { Cucum- } \\
\text { ber }\end{array}$} & T. harzianum & $16.6 \mathrm{gh}$ & $20.0 \mathrm{~g}$ & $26.6 \mathrm{f}$ & 30.0 ef & 30.0 ef & $26.6 \mathrm{f}$ & $33.3 \mathrm{e}$ & $33.3 \mathrm{e}$ & $33.3 \mathrm{e}$ & 27.7 \\
\hline & T. viride & $36.6 \mathrm{de}$ & $40.0 \mathrm{~d}$ & $33.3 \mathrm{e}$ & $36.6 \mathrm{de}$ & $40.0 \mathrm{~d}$ & $30.0 \mathrm{ef}$ & $33.3 \mathrm{e}$ & $36.6 \mathrm{de}$ & $40.0 \mathrm{~d}$ & 40.3 \\
\hline & B. subtilis & $26.6 \mathrm{f}$ & $33.3 \mathrm{e}$ & 30.0 ef & $26.6 \mathrm{f}$ & $33.3 \mathrm{e}$ & $23.3 \mathrm{fg}$ & $33.3 \mathrm{e}$ & 30.0 ef & $26.6 \mathrm{f}$ & 29.2 \\
\hline & P. fluorescence & $40.0 \mathrm{~d}$ & $46.6 \mathrm{c}$ & $40.0 \mathrm{~d}$ & $26.6 \mathrm{f}$ & $36.6 \mathrm{de}$ & $43.3 \mathrm{c}$ & $43.3 \mathrm{c}$ & $46.6 \mathrm{c}$ & $33.3 \mathrm{e}$ & 39.5 \\
\hline & S. serivisae & $46.6 \mathrm{c}$ & $50.0 \mathrm{bc}$ & $46.6 \mathrm{c}$ & $50.0 \mathrm{bc}$ & $46.6 \mathrm{c}$ & $46.6 \mathrm{c}$ & $43.3 \mathrm{c}$ & $43.3 \mathrm{c}$ & $40.0 \mathrm{~d}$ & 45.8 \\
\hline & Control & $53.3 \mathrm{~b}$ & $50.0 \mathrm{bc}$ & $50.0 \mathrm{bc}$ & $53.3 \mathrm{~b}$ & $46.6 \mathrm{c}$ & $46.6 \mathrm{c}$ & $50.0 \mathrm{bc}$ & $56.6 \mathrm{~b}$ & $50.0 \mathrm{bc}$ & 50.7 \\
\hline \multirow{6}{*}{$\begin{array}{l}\text { Canta- } \\
\text { loupe }\end{array}$} & T. harzianum & $20.0 \mathrm{~g}$ & $20.0 \mathrm{~g}$ & $26.6 \mathrm{f}$ & $23.3 \mathrm{fg}$ & $30.0 \mathrm{ef}$ & $26.6 \mathrm{f}$ & $23.3 \mathrm{fg}$ & $26.6 \mathrm{f}$ & 30.0 ef & 25.1 \\
\hline & T. viride & $33.3 \mathrm{e}$ & $36.6 \mathrm{de}$ & $33.3 \mathrm{e}$ & $43.3 \mathrm{c}$ & $40.0 \mathrm{~d}$ & $33.3 \mathrm{e}$ & $36.6 \mathrm{de}$ & 30.0 ef & $26.6 \mathrm{f}$ & 34.7 \\
\hline & B. subtilis & $23.3 \mathrm{fg}$ & $30.0 \mathrm{ef}$ & $26.6 \mathrm{f}$ & 30.0 ef & $26.6 \mathrm{f}$ & $33.3 \mathrm{e}$ & $30.0 \mathrm{ef}$ & $33.3 \mathrm{e}$ & 30.0 ef & 29.2 \\
\hline & P. fluorescence & $36.6 \mathrm{de}$ & $40.0 \mathrm{~d}$ & $46.6 \mathrm{c}$ & $33.3 \mathrm{e}$ & $40.0 \mathrm{~d}$ & $43.3 \mathrm{c}$ & $46.6 \mathrm{c}$ & $40.0 \mathrm{~d}$ & $33.3 \mathrm{e}$ & 39.9 \\
\hline & S. serivisae & $50.0 \mathrm{bc}$ & $40.0 \mathrm{~d}$ & $46.6 \mathrm{c}$ & $50.0 \mathrm{bc}$ & $36.6 \mathrm{de}$ & $40.0 \mathrm{~d}$ & $46.6 \mathrm{c}$ & $40.0 \mathrm{~d}$ & $36.6 \mathrm{de}$ & 42.9 \\
\hline & Control & $60.0 \mathrm{a}$ & $53.3 \mathrm{~b}$ & $50.0 \mathrm{bc}$ & $50.0 \mathrm{bc}$ & $43.3 \mathrm{c}$ & $50.0 \mathrm{bc}$ & $50.0 \mathrm{bc}$ & $53.3 \mathrm{~b}$ & $43.3 \mathrm{c}$ & 50.3 \\
\hline \multirow{6}{*}{ Tomato } & T. harzianum & $23.3 \mathrm{fg}$ & $23.3 \mathrm{fg}$ & $26.6 \mathrm{f}$ & $30.0 \mathrm{ef}$ & $26.6 \mathrm{f}$ & $23.3 \mathrm{fg}$ & $20.0 \mathrm{~g}$ & $26.6 \mathrm{f}$ & $23.3 \mathrm{fg}$ & 24.7 \\
\hline & T. viride & $36.6 \mathrm{de}$ & $33.3 \mathrm{e}$ & $33.3 \mathrm{e}$ & $40.0 \mathrm{~d}$ & $43.3 \mathrm{c}$ & $36.6 \mathrm{de}$ & $40.0 \mathrm{~d}$ & $33.3 \mathrm{e}$ & $33.3 \mathrm{e}$ & 36.6 \\
\hline & B. subtilis & $26.6 \mathrm{f}$ & $30.0 \mathrm{ef}$ & $23.3 \mathrm{fg}$ & $26.6 \mathrm{f}$ & $23.3 \mathrm{fg}$ & $33.3 \mathrm{e}$ & $26.6 \mathrm{f}$ & $33.3 \mathrm{e}$ & $23.3 \mathrm{fg}$ & 27.3 \\
\hline & P. fluorescence & $40.0 \mathrm{~d}$ & $43.3 \mathrm{c}$ & $50.0 \mathrm{bc}$ & $33.3 \mathrm{e}$ & $40.0 \mathrm{~d}$ & $40.0 \mathrm{~d}$ & $43.3 \mathrm{c}$ & $43.3 \mathrm{c}$ & $23.3 \mathrm{fg}$ & 39.1 \\
\hline & S. serivisae & $46.6 \mathrm{c}$ & $46.6 \mathrm{c}$ & $43.3 \mathrm{c}$ & $46.6 \mathrm{c}$ & $50.0 \mathrm{bc}$ & $43.3 \mathrm{c}$ & $40.0 \mathrm{~d}$ & $43.3 \mathrm{c}$ & $40.0 \mathrm{~d}$ & 44.4 \\
\hline & Control & $50.0 \mathrm{bc}$ & $53.3 \mathrm{~b}$ & $60.0 \mathrm{a}$ & $46.6 \mathrm{c}$ & $50.0 \mathrm{bc}$ & $53.3 \mathrm{~b}$ & $46.6 \mathrm{c}$ & $60.0 \mathrm{a}$ & $50.0 \mathrm{bc}$ & 51.0 \\
\hline \multirow{6}{*}{ Pepper } & T. harzianum & $20.0 \mathrm{~g}$ & $23.3 \mathrm{fg}$ & $26.6 \mathrm{f}$ & $23.3 \mathrm{fg}$ & $20.0 \mathrm{~g}$ & $23.3 \mathrm{fg}$ & $26.6 \mathrm{f}$ & 30.0 ef & $33.3 \mathrm{e}$ & 25.1 \\
\hline & T. viride & $40.0 \mathrm{~d}$ & $43.3 \mathrm{c}$ & $36.6 \mathrm{de}$ & $40.0 \mathrm{~d}$ & $40.0 \mathrm{~d}$ & $36.6 \mathrm{de}$ & $33.3 \mathrm{e}$ & $36.6 \mathrm{de}$ & $36.6 \mathrm{de}$ & 38.1 \\
\hline & B. subtilis & $23.3 \mathrm{fg}$ & $26.6 \mathrm{f}$ & $23.3 \mathrm{fg}$ & $26.6 \mathrm{f}$ & $23.3 \mathrm{fg}$ & $20.0 \mathrm{~g}$ & $23.3 \mathrm{fg}$ & $26.6 \mathrm{f}$ & $46.6 \mathrm{c}$ & 26.6 \\
\hline & P. fluorescence & $40.0 \mathrm{~d}$ & $43.3 \mathrm{c}$ & $40.0 \mathrm{~d}$ & $33.3 \mathrm{e}$ & $46.6 \mathrm{c}$ & $40.0 \mathrm{~d}$ & $43.3 \mathrm{c}$ & $46.6 \mathrm{c}$ & 30.0 ef & 40.3 \\
\hline & S. serivisae & $43.3 \mathrm{c}$ & $43.3 \mathrm{c}$ & $43.3 \mathrm{c}$ & $40.0 \mathrm{~d}$ & $46.6 \mathrm{c}$ & $43.3 \mathrm{c}$ & $46.6 \mathrm{c}$ & $46.6 \mathrm{c}$ & $40.0 \mathrm{~d}$ & 43.6 \\
\hline & Control & $53.3 \mathrm{~b}$ & $46.6 \mathrm{c}$ & $46.6 \mathrm{c}$ & $50.0 \mathrm{bc}$ & $50.0 \mathrm{bc}$ & $53.3 \mathrm{~b}$ & $50.0 \mathrm{bc}$ & $50.0 \mathrm{bc}$ & $50.0 \mathrm{bc}$ & 48.8 \\
\hline
\end{tabular}

Mean values within columns followed by the same letter are not significantly different $(\mathrm{P} \leq 0.05)$ 

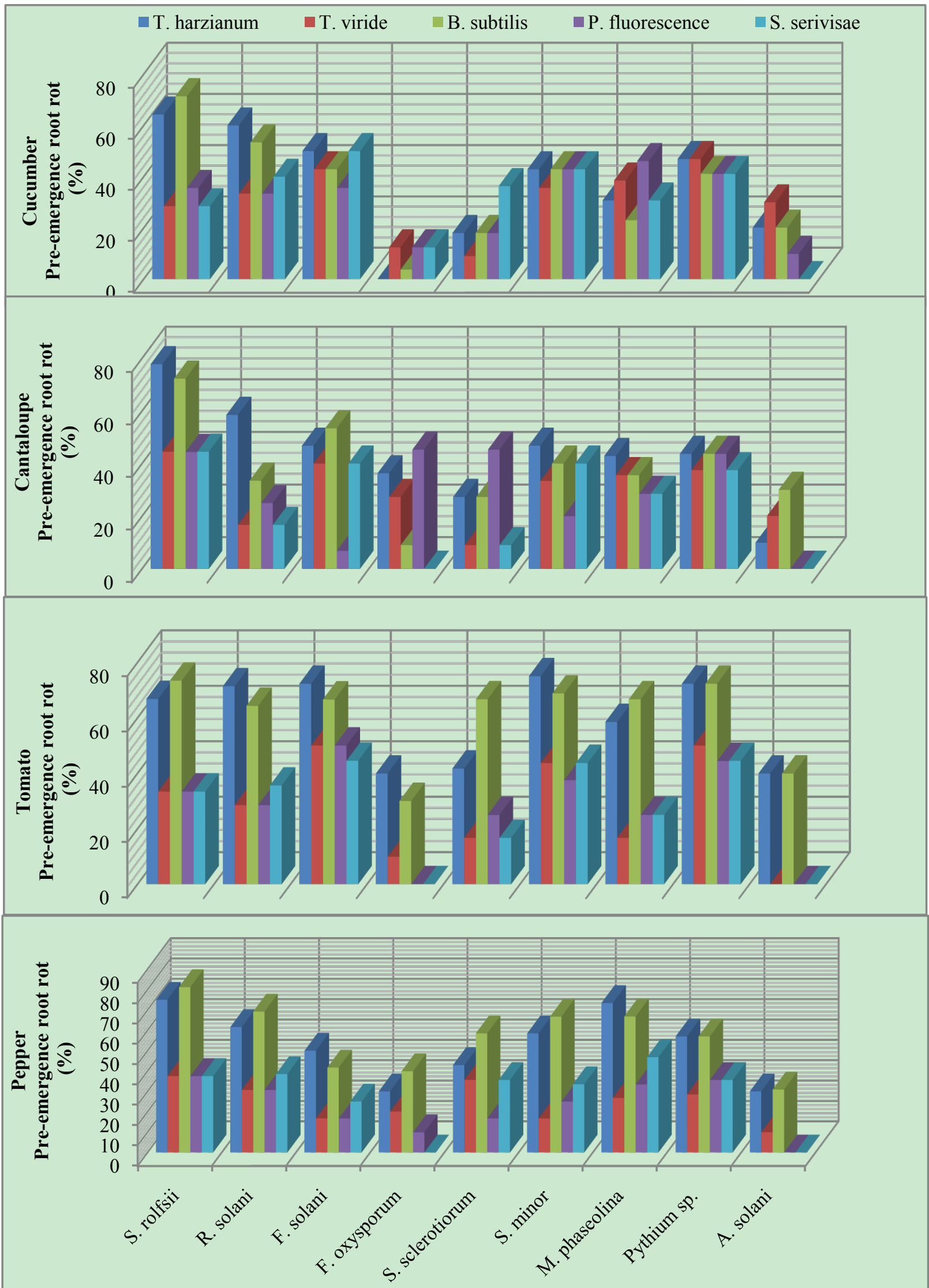

Figure 1. Reduction in vegetables root diseases vegetables root diseases at pre-emergence growth stage caused by soil-borne pathogenic fungi in response to applying antagonistic bio-agents as seed dressing under open greenhouse conditions 


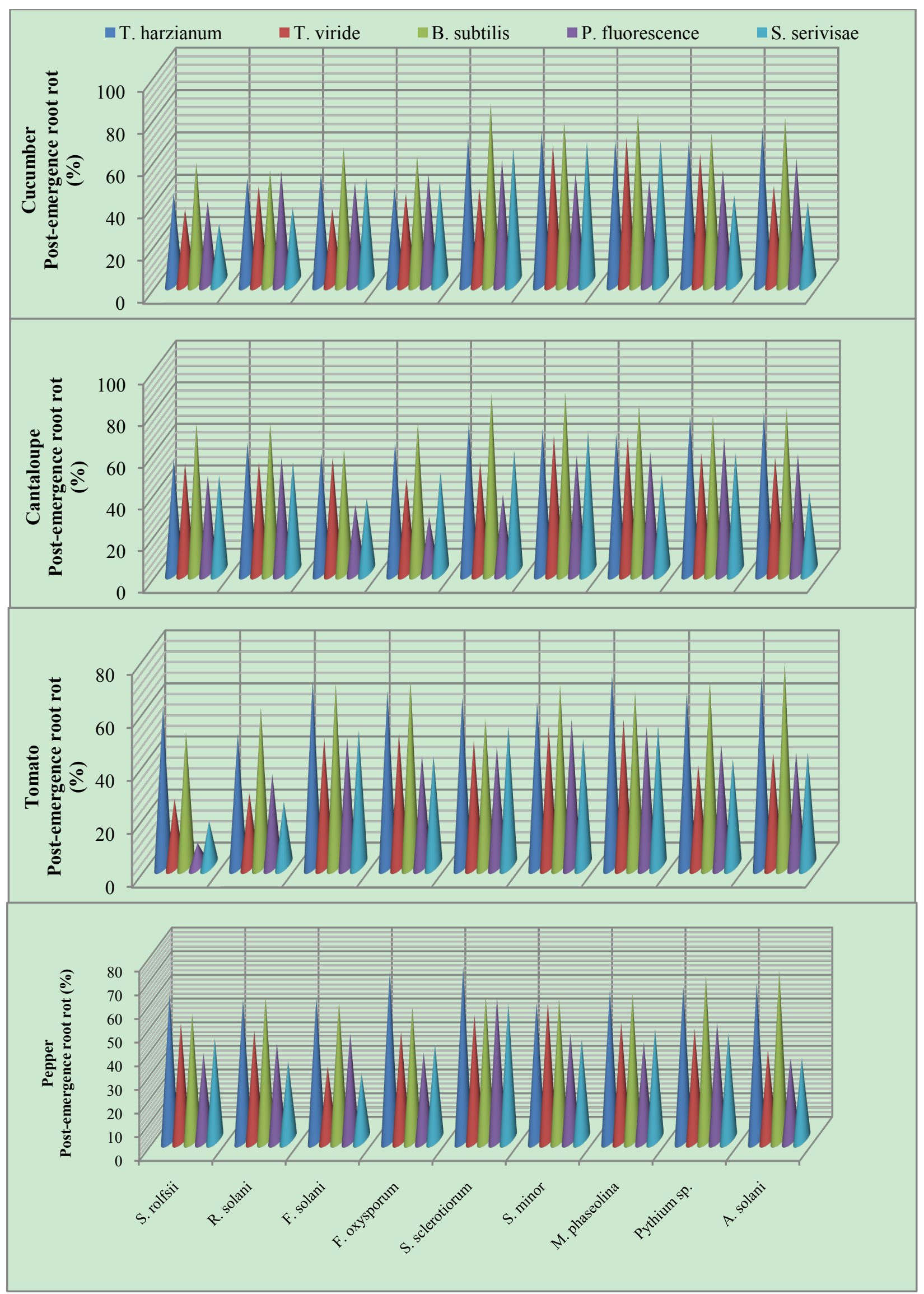

Figure 2. Reduction in vegetables root diseases vegetables root diseases at post-emergence growth stage caused by soil-borne pathogenic fungi in response to applying antagonistic bio-agents as seed dressing under open greenhouse conditions 
Table 4. Effect of applying antagonistic bio-agents as soil treatment against vegetables root diseases at post-emergence growth stage caused by soil-borne pathogenic fungi under open greenhouse conditions

\begin{tabular}{|c|c|c|c|c|c|c|c|c|c|c|c|}
\hline \multirow{3}{*}{ plant } & \multirow{3}{*}{ Bio-agent } & \multicolumn{10}{|c|}{ Pre-emergence root diseases incidence (\%) } \\
\hline & & \multicolumn{10}{|c|}{ Soil-borne pathogenic fungi } \\
\hline & & $\begin{array}{l}S . \\
\text { rolfsii }\end{array}$ & $\begin{array}{c}R . \\
\text { solani }\end{array}$ & $\begin{array}{c}F . \\
\text { solani }\end{array}$ & $\begin{array}{c}F . \\
\text { oxysporum }\end{array}$ & $\begin{array}{c}S . \\
\text { Sclero- } \\
\text { tiorum }\end{array}$ & $\begin{array}{c}S . \\
\text { minor }\end{array}$ & $\begin{array}{c}\text { M. } \\
\text { phaseolina }\end{array}$ & $\begin{array}{l}\text { Pythium } \\
\text { sp. }\end{array}$ & $\begin{array}{c}\text { A. } \\
\text { solani }\end{array}$ & Mean \\
\hline \multirow{6}{*}{ Cucumber } & T. harzianum & $0.0 \mathrm{j}$ & $0.0 \mathrm{j}$ & $27.7 \mathrm{i}$ & $0.0 \mathrm{j}$ & $0.0 \mathrm{j}$ & $0.0 \mathrm{j}$ & $0.0 \mathrm{j}$ & $0.0 \mathrm{j}$ & $0.0 \mathrm{j}$ & 3.0 \\
\hline & T. viride & $36.6 \mathrm{~h}$ & $55.5 \mathrm{ef}$ & $40.0 \mathrm{~g}$ & $47.3 \mathrm{~g}$ & $44.4 \mathrm{~g}$ & $33.3 \mathrm{~h}$ & $40.0 \mathrm{~g}$ & $42.1 \mathrm{~g}$ & $44.4 \mathrm{~g}$ & 42.6 \\
\hline & B. subtilis & $31.8 \mathrm{~h}$ & $0.0 \mathrm{j}$ & $0.0 \mathrm{j}$ & $0.0 \mathrm{j}$ & $0.0 \mathrm{j}$ & $0.0 \mathrm{j}$ & $0.0 \mathrm{j}$ & $0.0 \mathrm{j}$ & $0.0 \mathrm{j}$ & 3.5 \\
\hline & $\begin{array}{l}\text { P. fluores- } \\
\text { cence }\end{array}$ & 55.5 ef & $62.5 \mathrm{e}$ & $61.1 \mathrm{e}$ & $60.0 \mathrm{e}$ & $68.7 \mathrm{e}$ & $47.0 \mathrm{~g}$ & $64.7 \mathrm{e}$ & $78.7 \mathrm{~d}$ & $73.3 \mathrm{~d}$ & 63.5 \\
\hline & S. serivisae & $68.7 \mathrm{e}$ & $73.3 \mathrm{~d}$ & $53.3 \mathrm{f}$ & $81.8 \mathrm{c}$ & $62.5 \mathrm{e}$ & $52.5 \mathrm{f}$ & $53.3 \mathrm{f}$ & $52.9 \mathrm{f}$ & $50.0 \mathrm{f}$ & 60.9 \\
\hline & Control & $100 \mathrm{a}$ & $100 \mathrm{a}$ & $62.5 \mathrm{e}$ & $100 \mathrm{a}$ & $89.4 \mathrm{c}$ & $93.7 \mathrm{~b}$ & $100 \mathrm{a}$ & $84.6 \mathrm{c}$ & $80.0 \mathrm{c}$ & 90.0 \\
\hline \multirow{6}{*}{ Cantaloupe } & T. harzianum & $33.3 \mathrm{~h}$ & $37.5 \mathrm{~h}$ & $31.8 \mathrm{~h}$ & $0.0 \mathrm{j}$ & $0.0 \mathrm{j}$ & $0.0 \mathrm{j}$ & $0.0 \mathrm{j}$ & $0.0 \mathrm{j}$ & $0.0 \mathrm{j}$ & 11.4 \\
\hline & T. viride & $45.6 \mathrm{~g}$ & $42.1 \mathrm{~g}$ & $35.0 \mathrm{~h}$ & $52.9 \mathrm{f}$ & $44.4 \mathrm{~g}$ & $40.0 \mathrm{~g}$ & $52.6 \mathrm{f}$ & $38.0 \mathrm{~h}$ & $36.3 \mathrm{~h}$ & 42.9 \\
\hline & B. subtilis & $30.4 \mathrm{~h}$ & $0.0 \mathrm{j}$ & $27.2 \mathrm{i}$ & $38.0 \mathrm{~h}$ & $0.0 \mathrm{j}$ & $0.0 \mathrm{j}$ & $0.0 \mathrm{j}$ & $0.0 \mathrm{j}$ & $0.0 \mathrm{j}$ & 10.6 \\
\hline & $\begin{array}{l}\text { P. fluores- } \\
\text { cence }\end{array}$ & $52.6 \mathrm{f}$ & $55.5 \mathrm{ef}$ & $62.5 \mathrm{e}$ & $60.0 \mathrm{e}$ & $61.1 \mathrm{e}$ & $52.9 \mathrm{f}$ & $60.0 \mathrm{e}$ & $50.0 \mathrm{f}$ & $52.9 \mathrm{f}$ & 56.3 \\
\hline & S. serivisae & $66.6 \mathrm{e}$ & $78.5 \mathrm{~d}$ & $62.5 \mathrm{e}$ & $53.3 \mathrm{f}$ & $52.9 \mathrm{f}$ & $61.1 \mathrm{e}$ & $68.7 \mathrm{e}$ & $61.1 \mathrm{e}$ & $52.6 \mathrm{f}$ & 61.9 \\
\hline & Control & $83.3 \mathrm{c}$ & $94.4 \mathrm{~b}$ & $73.3 \mathrm{~d}$ & $90.0 \mathrm{~b}$ & $94.7 \mathrm{~b}$ & $100 \mathrm{a}$ & $93.7 \mathrm{~b}$ & $92.8 \mathrm{~b}$ & $80.0 \mathrm{c}$ & 89.1 \\
\hline \multirow{6}{*}{ Tomato } & T. harzianum & $34.7 \mathrm{~h}$ & $39.1 \mathrm{~h}$ & $27.7 \mathrm{i}$ & $33.3 \mathrm{~h}$ & $31.8 \mathrm{~h}$ & $39.1 \mathrm{~h}$ & $37.5 \mathrm{~h}$ & $36.3 \mathrm{~h}$ & $34.7 \mathrm{~h}$ & 34.9 \\
\hline & T. viride & $36.8 \mathrm{~h}$ & $45.0 \mathrm{~g}$ & $40.0 \mathrm{~g}$ & $44.4 \mathrm{~g}$ & $47.0 \mathrm{~g}$ & $52.6 \mathrm{f}$ & $55.5 \mathrm{ef}$ & $45.0 \mathrm{~g}$ & $50.0 \mathrm{f}$ & 46.2 \\
\hline & B. subtilis & $27.2 \mathrm{i}$ & $33.3 \mathrm{~h}$ & $30.4 \mathrm{~h}$ & $36.3 \mathrm{~h}$ & $34.7 \mathrm{~h}$ & $35.0 \mathrm{~h}$ & $31.8 \mathrm{~h}$ & $35.0 \mathrm{~h}$ & $26.0 \mathrm{i}$ & 32.1 \\
\hline & $\begin{array}{l}\text { P. fluores- } \\
\text { cence }\end{array}$ & $55.5 \mathrm{ef}$ & $58.8 \mathrm{ef}$ & $60.0 \mathrm{e}$ & $68.7 \mathrm{e}$ & $53.7 \mathrm{f}$ & $55.5 \mathrm{ef}$ & $64.7 \mathrm{e}$ & $52.9 \mathrm{f}$ & $60.0 \mathrm{e}$ & 58.8 \\
\hline & S. serivisae & $60.0 \mathrm{e}$ & $64.2 \mathrm{e}$ & $58.8 \mathrm{ef}$ & $62.5 \mathrm{e}$ & $60.0 \mathrm{e}$ & $70.5 \mathrm{~d}$ & $75.0 \mathrm{~d}$ & 58.8 ef & $50.0 \mathrm{f}$ & 62.2 \\
\hline & Control & $62.5 \mathrm{e}$ & $68.7 \mathrm{e}$ & $91.6 \mathrm{~b}$ & $85.0 \mathrm{c}$ & $94.4 \mathrm{~b}$ & $100 \mathrm{a}$ & $100 \mathrm{a}$ & $91.6 \mathrm{~b}$ & $86.9 \mathrm{c}$ & 86.7 \\
\hline \multirow{6}{*}{ Pepper } & T. harzianum & $25.0 \mathrm{i}$ & $34.7 \mathrm{~h}$ & $31.8 \mathrm{~h}$ & $34.7 \mathrm{~h}$ & $37.5 \mathrm{~h}$ & $34.7 \mathrm{~h}$ & $31.8 \mathrm{~h}$ & $38.0 \mathrm{~h}$ & $50.0 \mathrm{f}$ & 35.3 \\
\hline & T. viride & $44.4 \mathrm{~g}$ & $47.0 \mathrm{~g}$ & $47.3 \mathrm{~g}$ & $44.4 \mathrm{~g}$ & $44.4 \mathrm{~g}$ & $52.6 \mathrm{f}$ & $35.0 \mathrm{~h}$ & $36.8 \mathrm{~h}$ & $36.8 \mathrm{~h}$ & 43.1 \\
\hline & B. subtilis & $34.7 \mathrm{~h}$ & $36.3 \mathrm{~h}$ & $34.7 \mathrm{~h}$ & $31.8 \mathrm{~h}$ & $34.7 \mathrm{~h}$ & $37.5 \mathrm{~h}$ & $34.7 \mathrm{~h}$ & $27.2 \mathrm{i}$ & $50.0 \mathrm{f}$ & 35.7 \\
\hline & $\begin{array}{c}\text { P. fluorescen- } \\
\text { ce }\end{array}$ & $44.4 \mathrm{~g}$ & $47.0 \mathrm{~g}$ & $50.0 \mathrm{f}$ & $66.6 \mathrm{e}$ & $66.6 \mathrm{e}$ & $64.2 \mathrm{e}$ & $47.0 \mathrm{~g}$ & $50.0 \mathrm{f}$ & $66.6 \mathrm{e}$ & 55.8 \\
\hline & S. serivisae & $58.8 \mathrm{ef}$ & $62.5 \mathrm{e}$ & $52.9 \mathrm{f}$ & $50.0 \mathrm{f}$ & $62.5 \mathrm{e}$ & $58.8 \mathrm{ef}$ & $56.2 \mathrm{ef}$ & $60.0 \mathrm{e}$ & $55.5 \mathrm{ef}$ & 57.4 \\
\hline & Control & $78.5 \mathrm{~d}$ & $88.2 \mathrm{c}$ & $72.2 \mathrm{~d}$ & $75.0 \mathrm{~d}$ & $100 \mathrm{a}$ & $94.4 \mathrm{~b}$ & $93.3 \mathrm{~b}$ & $93.7 \mathrm{~b}$ & $76.1 \mathrm{~d}$ & 85.7 \\
\hline
\end{tabular}



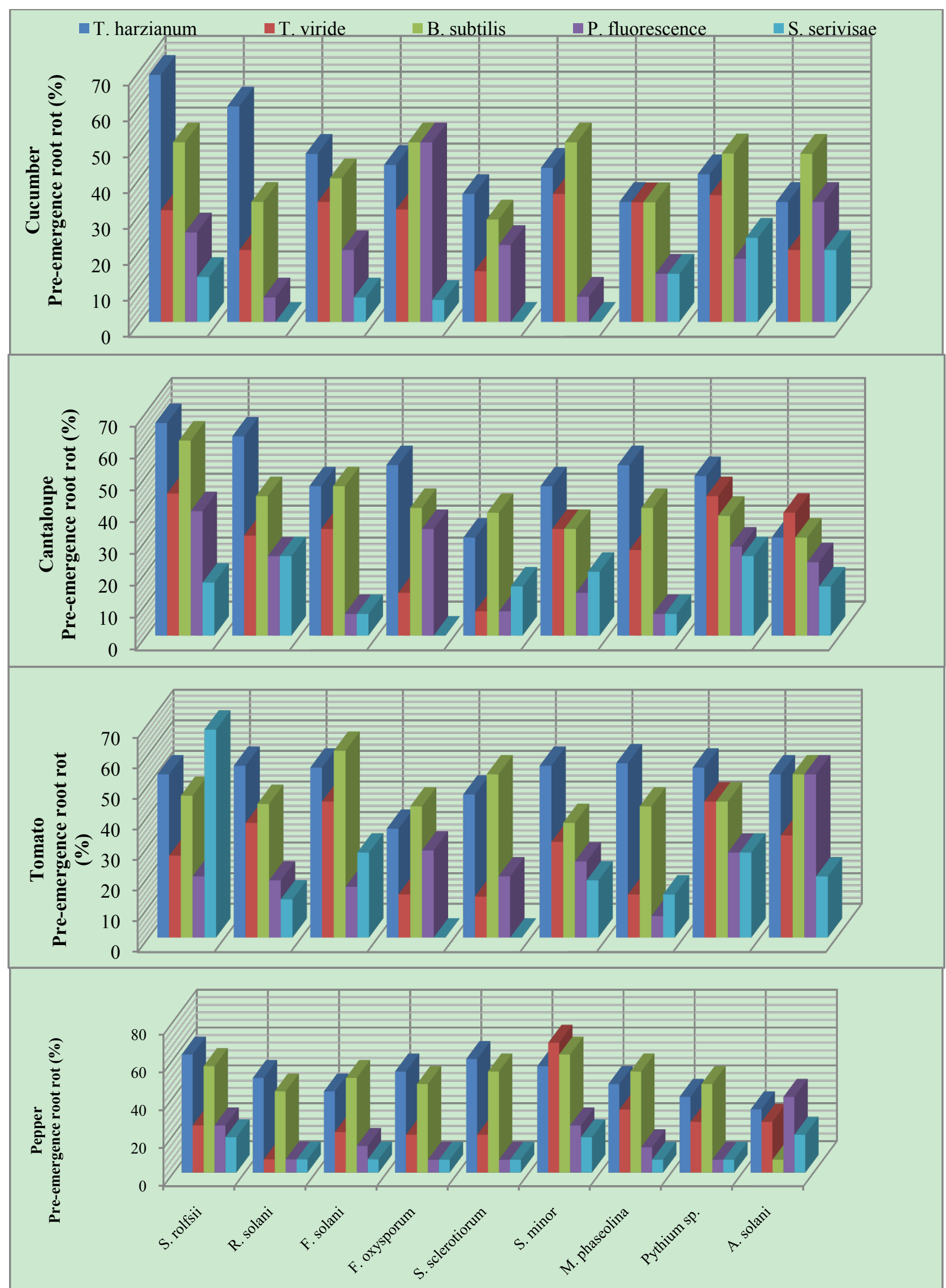

Figure 3. Reduction in vegetables root diseases vegetables root diseases at pre-emergence growth stage caused by soil-borne pathogenic fungi in response to applying antagonistic bio-agents as soil treatment under open greenhouse conditions 


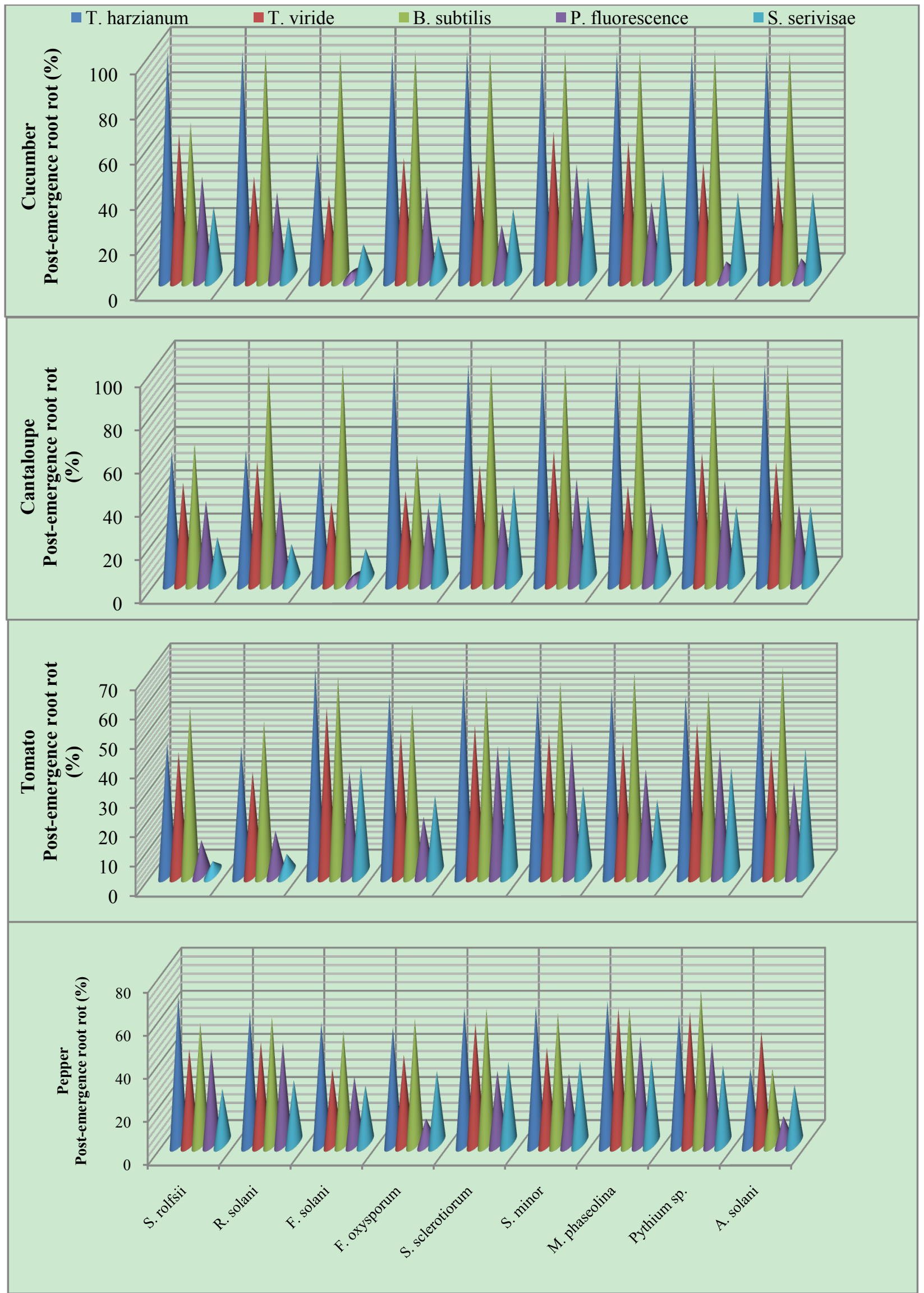

Figure 4. Reduction in vegetables root diseases vegetables root diseases at post-emergence growth stage caused by soil-borne pathogenic fungi in response to applying antagonistic bio-agents as soil treatment under open greenhouse conditions 
Furthermore, for the effective biological control of soilborne plant pathogens, a major consideration has been given to proliferation of the antagonist after introduction into the soil. Among the desirable attributes of a successful antagonist is its ability to produce inoculum in excess and to survive, grow, and proliferate in soil and the rhizosphere[27]. Various actinomycetes, bacteria, and fungi, which show antagonism to $P$. capsici, exist in soils where peppers are grown [28-30]. In particular, some antagonistic rhizobacteria such as Burkholderia cepacia[29], and Pseudomonas aeruginosa [30] very effective against Phytophthora blight in pepper plants under laboratory and greenhouse conditions. Similarly, application of B. cepacia granules into soil provided better suppression of Phytophthora blight on red-pepper seedlings, as compared to direct drenching with Burkholderia cepacia suspensions[31]. Soil drenches and dipping of seedling roots with the antagonist suspensions were found to be more effective in disease suppression than the coating and dipping pepper seeds[29]. Also,[32] reported that Trichoderma are present in all soil and they are the most cultural fungi. Trichoderma species are strongly antagonistic to other phytopathogenic fungi. They produce hydrolytic enzymes which are believed to play an important role in the parasitism of phytopathogenic fungi.

The present study demonstrate that the use of bio-agent treatments, B. subtilis, P. flourescens and S. serevisiae either as seed soaking or soil drench could reduce root roit incidence of tested vegetables grown in soil artificially infested with disease incidents. These results are in agreements with previous reports in this concern[24,33]. Several workers explained the mode of action of antagonistic bacterial and yeast isolates. In this regards, the potential of Bacillus sp. to synthesise a wide variety of metabolites with antifungal activity is known and in recent years it has been a subject of experiments[34]. Most of these substances belong to lipopeptides, especially from surfactin, iturin and fengicin classes. Not so much is known about the mechanism of antifungal activity of these substances produced by Bacillus sp. Some of them (iturin and surfactin) are able to modify bacterial surface hydrophobicity and, consequently, microbial adhesion to surfaces (to mycelium)[34]. Antibiotics of the iturin group were found to act upon the sterol present in the cytoplasmic membrane of the fungi[35]. Biological control of Aspergillus niger by Bacillus subtilis was also investigated[36]. They demonstrated that the bacterial cells initially adhered to the fungus, multiplied and extensively colonised the surface. Rapid growth of bacterial cells on the surface resulted in damage of fungal cell walls. These aspects appear essential in association with the antifungal properties of Bacillus sp. used in the biological control of plant diseases. Moreover, although the biocontrol activity of antagonistic bacteria and yeasts has been demonstrated on a variety of commodities, the mode of action of these microbial biocontrol agents has not been fully elucidated. In the case of bacterial antagonists, it has been suggested that their biocontrol activity may be partly associated with the production of antibiotics[37,38]. Antagonistic yeasts have been selected mainly for their capability to rapidly colonize and grow in surface wounds, and subsequently to out compete the pathogen for nutrients and space. Competition for nutrients and space is believed to be the major component of the mode of action of antagonistic yeasts[39,40]. Besides competing for nutrients and space, antagonistic yeasts parasitize major postharvest pathogens directly, through strong attachment to their hyphae. This leads to partial disruption of hyphal wall structures[40].

In conclusion, vegetable crops are not spared from destruction by fungal pathogens, which infect roots, stems, leaves, flowers and fruits. In addition, fungi can infect the roots and colonize the vascular tissues of the plant, causing root rots and wilt diseases Most plant pathogens affecting vegetable crop species have been reasonably well-studied and information on their biology is available[41]. The use of fungi, bacteria and yeasts to manage these diseases requires disruption of some stage of the disease or life cycle of the pathogen and this has been achieved through several different mechanisms. Prevention of infection, reduction in colonization of host tissues, or reducing sporulation or survival of the pathogen, can each provide a level of disease control using biological control agents. Therefore, he objective of the present study was to evaluate the different approaches activity of some antagonistic fungal and bacterial agents against vegetables root rot incidence when applied as seed treatment or soil drench under open greenhouse conditions. On this point of view, the present study of demonstrates that application of bio-agents as seed treatment and soil drench may be useful for controlling root rot disease in field.

\section{ACKNOLEDGMENTS}

This work was supported financially by the Science and Technology Development Fund (STDF), Egypt, Grant No. 1059.

\section{REFERENCES}

[1] Sivan, A., 1987, Biological control of Fusarium crown rot of tomato by Trichoderma harzianum under field conditions. Plant Dis., 71, 587-592. Doi: 10.1094/PD-71-0587.

[2] Abdel-Kader, M.M., 1997, Field application of Trichoderma harzianum as biocide for control of bean root rot disease. Egypt. J. Phytopathol., 25, 19-25.

[3] Sivan, A., Chet, I., 1986, Biological control of Fusarium spp. in cotton, wheat and muskmelon by Trichoderma harzianum. J. Phytopathol., 116, 39-47. Doi: 10.1111/j.1439-0434.1986. tb00892.x.

[4] Whipps, J.M., Lumsden, R.D., 2001, Commercial use of fungi as plant disease biological control agents: status and prospects. p. 9-22. In: "Fungi as Biocontrol Agents: Progress, Problems and Potential" (T.M. Butt, C. Jackson, N. Magan, 
eds.), (2001), CABI Publishing: Wallingford, UK.

[5] McLean, K.L., Dodd, S.L., Sleight, B.E., Hill, R.A., Stewart, A., 2004, Comparison of the behavior of a transformed hygromycin resistant strain of Trichoderma atoviride with the wild-type strain. NZ Plant Protect., 57, 72-76.

[6] Hadar, Y., Chet, I., Henis, Y., 1979, Biological control of $R$. solani damping-off with wheat bran culture of Trichoderma harzianum. Phytopathology, 69, 64-68.

[7] Hadar, Y., Harman, G.E., Taylor, A.G., 1984, Evaluation of Trichoderma koningii and T. harzianum from New York soils for biological control of seed rot caused by Pythium spp. Phytopathology, 74, 106-110.

[8] Elad, T., Chet, J., Katan, J., 1980, Trichoderma harzianum: a biocontrol effective against Sclerotium rolfsii and Rhizoctonia solani. Phytopathology, 70, 119-121.

[9] Kim, D.S., Cook, R.J., Weller, D.M. 1997, Bacillus sp. L324-92 for biological control of three root diseases of wheat grown with reduced tillage. Phytopathology, 87,551-558.

[10] Parke, J.L., Rand, R.E., Joy, A.E., King, E.B., 1991, Biological control of Pythium damping-off and Aphanomyces root rot of peas by application of Pseudomonas cepacia or $P$. fluorescens to seed. Plant Disease, 75,987-992.

[11] King, E.B., Parke, J.L., 1993. Biocontrol of Aphanomyces root rot and Pythium damping-off by Pseudomonas cepacia AMMD on four pea cultivars. Plant Disease, 77, 1185-1188.

[12] Callan, N.W., Mathre, D.E., Miller, J.B., 1990, Bio-priming seed treatment for biological control of Pythium ultimum pre-emergence damping-off in sh 2 sweet corn. Plant Disease, $74,368-372$.

[13] Baird, R.E., Nankam, C., Moghaddam, P.F., Pataky, J., 1994,. Evaluation of seed treatments on Shrunken-2 sweet corn. Plant Disease, 78, 817-821.

[14] Howell, C.R., Stepanovic, R.D., 1995,. Michanism in the biocontrol of Rhizoctonia solani - induced cotton seedling disease by Gliocladium virens: Antibiosis. Phytopathology, $85,469-472$.

[15] Mathre, D.E., Johnston, R.H., Callan, N.W., Mohan, S.K., Martin, J.M., Miller, J.B., 1995, Combined biological and chemical seed treatments for control of two seedling diseases of sh2 sweet corn, Plant Disease, 79, 1145-1148

[16] Abdel-Kader, M.M., 1999, Biological and chemical control of wilt disease of hot pepper (Capsicum annum L.). Egypt. J. Phytopathol., 27, 1-8.

[17] El-Mougy, N.S., Abdel-Kader, M.M., Abdel-Kareem, F., Embaby, E.I., El-Mohamady, R., Abd El-Khair, H., 2011, Survey of Fungal Diseases Affecting Some Vegetable Crops and Their Rhizospheric Soilborne Microorganisms Grown under Protected Cultivation System in Egypt. Research Journal of Agriculture and Biological Sciences, 7,(2), 203-211.

[18] Abd-Alla, M.A., El-Mohamedy, R.S.R., El-Mougy, N.S., 2007, Control of Sour Rot Disease of Lime Fruits Using Saprophytic Isolates of Yeast. Egypt. J. Phytopathol., 35, (2), 39-51.

[19] SAS, Institute Inc., 'SAS/STAT user's guide. Version 6. Vol. 2.' 12th edn. (SAS Institute Inc.: Cary, NC), (1996), 846 pp.
[20] Winer, B.J., 'Statistical principles in experimental design.' 2nd edn. (McGraw-Hill Kogakusha Ltd: Tokyo), (1971), 596 pp.

[21] Papavizas, G.C., 1982, Survival of Trichoderma harzianum in soil and in pea and bean rhizospheres. Phytopathology, 72, 121-125.

[22] Monte, E., 2001, Understanding Trichoderma: between biotechnology and microbial ecology. Int. Microbiol., 4,1- 4.

[23] Chao W.L., Nelson E.B., Harman G.E., Hoch H.C. 1986. Colonization of the rhizosphere by biological control agents applied to seeds. Phytopathology 76: 60-65.

[24] El-Mougy, N.S., 2001, Field application of certain biological and chemical approaches on controlling Bean wilt disease. Egypt. J. Phytopathol., 29, 69-78.

[25] Wright, B., Rowse, H.R., Whipps, J.M., 2003, Application of beneficial microorganisms to seeds during drum priming. Biocontrol Sci. Technol., 13, 599-614. Doi: 10.1080/095831 50310001517992

[26] El-Mougy, N.S., Abdel-Kader, M.M., 2008, Long term activity of bio-priming seed treatment for biological control of faba bean root rot pathogens. Aust. Plant Pathol., 37, (5), 464-471.

[27] Baker, K.F., Cook, R.J., 1974, Biological control of plant pathogens. W. H. Freeman \& Co., San Francisco, CA, USA., (1974).

[28] Ahn, S.J., Hwang, B.K., 1992, Isolation of antibiotic- producing actinomycetes antagonistic to Phytophthora capsici from pepper-growing soils. Korean J. Mycol., 20,259-268.

[29] Jee, H.J., Nam, C.G., Kim, C.H., 1988, Studies on biological control of Phytophthora blight of red-pepper. I. Isolation of antagonists and evaluation of antagonistic activity in vitro and in greenhouse. Korean J. Plant Pathol., 4,305-312.

[30] Kim, B.S., Hwang, B.K., 1992, Isolation of antibiotic- producing bacteria antagonistic to Phytophthora capsici from pepper growing soils and evaluation of their antibiotic activity. Korean J. Plant Pathol., 8,241-248.

[31] Park, K.S., Jang, S.W., Kim, C.H., Lee, E.J., 1989, Studies on biological control of Phytophthora blight of red-pepper. III. Formulations of Trichoderma harzianum and Pseudomonas cepacia antagonistic to Phytophthora capsici and their preservation. Korean J. Plant Pathol., 5,131-138.

[32] Gomathinayagam, S., Rekha, M., Murugan, S., Jagessar, J.C., 2010, The biological control of paddy disease brown spot (Bipolaris oryzae) by using Trichoderma viride in vitro condition. Journal of Bio. pesticides, 3, 93-95.

[33] Peighami-Ashnaei, S., Sharifi-Tehrani, A., Ahmadzadeh, M., Behboudi, K., 2009, Interaction of different media on production and biocontrol efficacy of Pseudomonas fluorescens P-35 and Bacillus subtilis B-3 against grey mould of apple. Journal of Plant Pathology, 91, (1), 65-70.

[34] Ahimou, F., Jacques, P., Deleu, M., 2000, Surfactin and iturin A effects on Bacillus subtilis surface hydrophobicity. Enz. Microb. Technol., 27, 749-752.

[35] Worthington, P.A., 1988, Antibiotics with antifungal and antibacterial activity against plant diseases. Nat. Prod. Rep., 5, 47-50. 
[36] Podile, A.R., Parkash, A.P., 1996, Lysis and biological con trol of Aspergillus niger by Bacillus subtilis AF 1. Can. J. Microbiol., 42, 533-537.

[37] Bull, C.T., Stack, J.P., Smilanick, J.L., 1997, Pseudomonas syringae strains ESC-10 and ESC-11 survive in wounds on citrus and control green and blue molds of citrus. Biol. Control, $8,81-88$.

[38] Janisiewicz, W., Yourman, L., Roitman, J., Mahoney, N., 1991, Postharvest control of blue mold and gray mold of apples and pears by dip treatment with pyrrolnitrin, a metabolite of Pseudomonas cepacia. Plant Dis., 75, 490-494.
[39] El-Ghaouth, A., Wilson, C. L., Wisniewski, M., 1998, Ultrastructural and cytochemical aspects of the biological control of Botrytis cinerea by Candida saitoana in apple fruit. Phytopathology, 88, 282-291.

[40] Wisniewski, M., Biles, C., Droby, S., McLaughlin, R., Wilson, C., Chalutz, E., 1991, Mode of action of the postharvest biocontrol yeast, Pichia guilliermondii. I. Characterization of the attachment to Botrytis cinerea. Physiol. Mol. Plant Pathol., $39,245-258$.

[41] Agrios, G.N., 1997. Plant Pathology, 4th edn, Academic Press, (1997). 